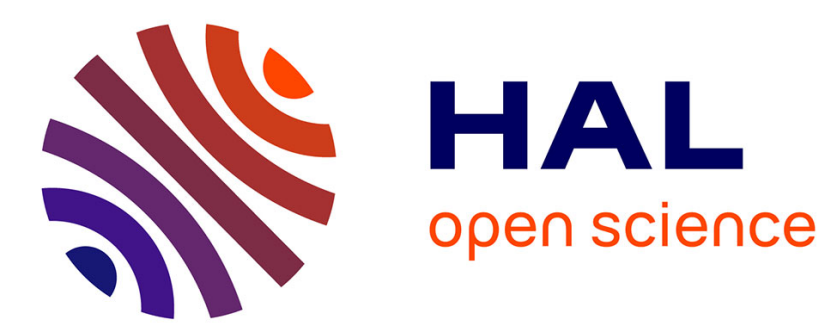

\title{
CARACTÉRISATION DE SOURCES EN ACOUSTIQUE SOUS-MARINE
}

S. Bourennane, Jean-Baptiste Faure

\section{To cite this version:}

S. Bourennane, Jean-Baptiste Faure. CARACTÉRISATION DE SOURCES EN ACOUSTIQUE SOUS-MARINE. Journal de Physique Colloques, 1990, 51 (C3), pp.C3-23-C3-31. 10.1051/jphyscol:1990303 . jpa-00230731

\section{HAL Id: jpa-00230731 https://hal.science/jpa-00230731}

Submitted on 1 Jan 1990

HAL is a multi-disciplinary open access archive for the deposit and dissemination of scientific research documents, whether they are published or not. The documents may come from teaching and research institutions in France or abroad, or from public or private research centers.
L'archive ouverte pluridisciplinaire HAL, est destinée au dépôt et à la diffusion de documents scientifiques de niveau recherche, publiés ou non, émanant des établissements d'enseignement et de recherche français ou étrangers, des laboratoires publics ou privés. 


\title{
CARACTÉRISATION DE SOURCES EN ACOUSTIQUE SOUS-MARINE
}

\author{
S. BOURENNANE et B. FAURE \\ CEPHAG, CNRS UA-346, ENSIEG, BP 46, F-38402 st-Martin d'Hères Cedex, \\ France
}

Résumé : Pour caractériser des sources rayonnantes large bande à partir des signaux reçus sur un réseau de capteurs, plusieurs méthodes ont été développées à ce jour. Les solutions proposées sont essentiellement basées sur des moyennes temporelles, spatiales et/ou fréquentielles. Dans cette étude nous regroupons ces méthodes en deux principales catégories (méthodes à sous-espace signal incohérent et méthodes à sous-espace signal cohérent). La deuxième approche de ces méthodes nécessite le calcul des opérateurs, dits de focalisation. La construction de ces opérateurs nécessite le choix des paramètres de focalisation. Nous proposons dans cette étude un opérateur de focalisation et nous donnons des régles de contrôle permettant de choisir des valeurs optimales de ces différents paramètres.

Ces diverses méthodes de traitement d'antenne large bande sont appliquées à des signaux expérimentaux reçus sur une antenne en acoustique sous marine.

\begin{abstract}
Several methods have been proposed to caracterize the radiating wideband sources from the received signals on an antenna. The proposed methods are based in the temporal, spatial and/or frequential smoothing. In this paper, we regroup these methods into two principal categories : the incoherent signal subspace methods and the coherent signal subspace methods. The second approach of these methods uses the focusing operators. The construction of these operators needs the choice of the focusing parameters. In this study we propose a new focusing operator and we show the importance of the choice of the focusing parameters in practice.

The different wideband array processing methods are applied on the underwater experimental data.
\end{abstract}

\section{1- INTRODUCTION}

Dans cette étude, nous nous intéressons à la localisation de sources large bande. Nous regroupons les différentes méthodes existantes en deux principales catégories : les méthodes à sous-espace signal incohérent et les méthodes à sous espace signal cohérent. Dans cette deuxième catégorie de méthodes nous proposons un nouvel opérateur de focalisation permettant de corriger les matrices interspectrales estimées aux différentes fréquences de la bande, de manière à pouvoir les moyenner d'une façon cohérente. Nous montrons l'importance du choix des paramètres intervenant lors de la construction de l'opérateur de focalisation.

Nous présentons des résultats obtenus à partir de signaux expérimentaux et nous dégageons des limites d'applications de ces méthodes.

\section{2 - POSITION DU PROBLEME}

\section{2-1 Matrice interspectrale}

On considère l'observation vectorielle $r(t)=\left[r_{1}(t), \ldots, r_{N}(t)\right]^{T}$ mesurée sur les sorties des $N$ capteurs d'une antenne pendant une durée $T$; où $r_{i}(t)$ représente le signal reçu sur le capteur i :

$$
r_{i}(t)=\sum_{k=1}^{p} \alpha_{i_{k}} S_{k}\left(t-\tau_{i_{k}}\right)+b_{i}(t) \quad-T / 2 \leq t \leq T / 2
$$


$\mathrm{S}_{\mathrm{k}}($.$) : le signal émis par la \mathrm{k}^{\mathrm{ième}}$ source; $\tau_{\mathrm{i}}$ : la durée de propagation source $\mathrm{k} /$ capteur $\mathrm{i}$; $b_{i}(t)$ : bruit additif sur le capteur $i ; \alpha_{i_{k}}$ : amplitude complexe du signal émis par la source $k$ et reçue sur le capteur i.

En se plaçant dans les hypothèses classiques du traitement d'antenne [1] ; $r_{i}(t)$ s'écrit dans le domaine fréquentiel (avec $\alpha_{i_{k}}=\alpha_{i}=1$ ).

$$
R_{i}\left(f_{n}\right)=\sum_{k=1}^{P} S_{k}\left(f_{n}\right) e^{-j 2 \pi f_{n} \tau_{i_{k}}+B_{i}\left(f_{n}\right)}
$$

les fréquences $f_{n}(1 \leq n \leq M)$ sont espacées de $1 / T$.

En utilisant la notation matricielle, on obtient :

où

$$
\underline{R}\left(f_{n}\right)=\underline{\underline{A}}\left(f_{n}\right) \underline{S}\left(f_{n}\right)+\underline{B}\left(f_{n}\right)
$$

$\underline{\underline{A}}\left(f_{n}\right)=\left[\underline{a}_{1}\left(f_{n}\right), \ldots, \underline{a}_{p}\left(f_{n}\right)\right]$ est la matrice de transfert entre les $P$ sources et les $N$ capteurs de l'antenne à la fréquence $f_{n}$ avec : $\underline{a}_{k}\left(f_{n}\right)=\frac{1}{\sqrt{N}}\left[1, e^{-j \varphi_{k n}, . ., e^{-j(N-1)} \varphi_{k n}}\right]^{T}$ qui représente le vecteur directionnel de la source $k$.

La matrice interspectrale des signaux reçus à la fréquence $f_{n}$ est :

$$
\underline{\underline{\gamma}}\left(f_{n}\right)=\operatorname{cov}\left[\underline{R}\left(f_{n}\right)\right]=\underline{\underline{A}}\left(f_{n}\right) \underline{\underline{\gamma}}_{s}\left(f_{n}\right) \underline{\underline{A}}^{+}\left(f_{n}\right)+\underline{\underline{\gamma}}_{B}\left(f_{n}\right)
$$

Si de plus les $B_{i}\left(f_{n}\right)(1 \leq i \leq N)$ sont non corrélés et de même variance $\sigma^{2}\left(f_{n}\right)$

$$
\underline{\underline{\gamma}}\left(f_{n}\right)=\underline{\underline{A}}\left(f_{n}\right) \underline{\underline{y}}_{s}\left(f_{n}\right) \underline{\underline{A}}^{+}\left(f_{n}\right)+\sigma^{2}\left(f_{n}\right) \underline{\underline{I}} \quad 1 \leq n \leq M
$$

\section{2-2 Définition de l'opérateur de focalisation}

Dès que les différentes matrices sont estimées, il existe deux manières de les exploiter :

- soit nous les traitons séparément, puis nous moyennons les différents résultats,

- soit nous les moyennons, puis nous effectuons les traitements sur la matrice interspectrale moyenne.

Pour calculer la matrice interspectrale moyenne, nous sommes amenés à construire des opérateurs de focalisation permettant de corriger les différentes matrices interspectrales de manière à les rendre cohérentes pour conserver les informations concernant les directions des sources après l'opération de moyenne.

La matrice interspectrale moyenne est définie par :

$$
\hat{\underline{\underline{Y}}}\left(f_{0}\right)=\frac{1}{M} \sum_{n=1}^{M} \underline{\underline{H}}\left(f_{0}, f_{n}\right) \underline{\underline{Y}}\left(f_{n}\right) \underline{\underline{H}}^{+}\left(f_{0}, f_{n}\right)
$$

où $\underline{\underline{H}}\left(f_{0}, f_{n}\right)$ est l'opérateur de focalisation qui vérifie la condition [2] :

$$
\begin{aligned}
& \underline{\mathbf{H}}\left(\mathrm{f}_{0}, \mathrm{f}_{\mathrm{n}}\right) \cdot \underline{\underline{\mathbf{A}}}\left(\mathrm{f}_{\mathrm{n}}\right)=\underline{\underline{\mathbf{A}}}\left(\mathrm{f}_{0}\right) \quad \forall \mathrm{f}_{\mathrm{n}} \in \mathrm{L} \\
& \overline{\overline{(N} \times N)}\left(\begin{array}{l}
\mathrm{N} \times P) \\
(\mathrm{N} \times P)
\end{array}\right.
\end{aligned}
$$


$f_{0}$ est la fréquence de focalisation et $L$ est la largeur de la bande d'analyse.

\section{2-3 Construction de l'opérateur de focalisation}

Compte tenu de l'hypothèse classique en traitement d'antenne, $\mathrm{P}<\mathrm{N}$, le système ci-dessus est sous-déterminé ; par conséquent, pour construire $\underline{\underline{H}}\left(f_{0}, f_{n}\right)$, plusieurs approches ont été proposées [2], [3], [4], [1]. Certaines solutions nécessitent une connaissance a priori des directions des sources, d'autres par contre peuvent être obtenues sans connaissance a priori de ces directions.

-L'opérateur de focalisation que nous proposons est estimé à partir des vecteurs propres des matrices interspectrales estimées à chaque fréquence de la bande d'analyse L [1].

L'opérateur doit satisfaire :

1) $\underline{\underline{\mathbf{H}}}\left(\mathrm{fo}_{0}, \mathrm{fn}_{\mathrm{n}}\right) \underline{\underline{\mathrm{V}}}\left(\mathrm{f}_{\mathrm{n}}\right)=\underline{\underline{\mathrm{V}}}\left(\mathrm{fo}_{0}\right)$

2) $\underline{\underline{\underline{H}}}\left(f_{0}, f_{n}\right) \underline{\underline{\underline{H}}}^{+}\left(f_{0}, f_{n}\right)=\underline{\underline{I}}$

L'opérateur (NxN) qui vérifie ces conditions est donc [1] :

$$
\underline{\underline{H}}\left(f_{0}, f_{n}\right)=\underline{\underline{V}}\left(f_{0}\right) \underline{\underline{V}}^{+}\left(f_{n}\right) \quad \forall f_{n} \in L
$$

Cet opérateur a l'avantage de transformer la base orthonormale des vecteurs propres engendrant le sous-espace signal à la fréquence $f_{n}$ en la base orthonormale des vecteurs propres engendrant le sous-espace signal à la fréquence $f_{0}$. Cette conservation des angles préserve au mieux l'écart entre les différentes sources.

\section{3 - CHOIX DES PARAMETRES DE FOCALISATION}

La construction d'opérateurs de focalisation nécessite le choix de la fréquence de focalisation, la largeur de la bande à analyser et les directions de focalisation. En général, les angles de focalisation sont donnés par une première analyse spatiale.

Considérons le cas où une seule direction initiale est trouvée, l'opérateur de focalisation est une matrice diagonale [2] : soit $\hat{\theta}$ la direction de focalisation, $\theta_{i}(i=1, \ldots, P)$ les directions des $P$ sources rayonnantes. Nous avons à chaque fréquence $f_{n}(n=1, \ldots, M)$ le vecteur directionnel de la source i :

où

$$
\underline{a}_{\theta_{i}}\left(f_{n}\right)=\frac{1}{\sqrt{N}}\left[1, e^{-j \varphi\left(\theta_{i}, f_{n}\right)}, \ldots, e^{-j(N-1) \varphi\left(\theta_{i}, f_{n}\right)}\right]^{T}
$$

$\varphi\left(\theta_{i}, f_{n}\right)=2 \pi f_{n} \frac{d}{c} \sin \theta_{i}$

(d est l'espacement entre capteurs et c est la célérité du son dans le milieu).

Après focalisation, ce vecteur devient: $\quad \underline{a}^{\prime} \theta_{i}\left(f_{0}, f_{n}\right)=\underline{\underline{H}}\left(f_{0}, f_{n}\right) \underline{a} \theta_{i}\left(f_{n}\right)$

où soit :

$$
\underline{\underline{H}}\left(\mathrm{f}_{0}, \mathrm{f}_{\mathrm{n}}\right)=\operatorname{diag}\left[1, \mathrm{e}^{-\mathrm{j} 2 \pi\left(\mathrm{f}_{0}, \mathrm{f}_{\mathrm{n}}\right) \sin \hat{\theta}}, \ldots, \mathrm{e}^{-\mathrm{j}(\mathrm{N}-1) 2 \pi\left(\mathrm{f}_{0}, \mathrm{f}_{n}\right) \sin \hat{\theta}}\right]
$$




$$
\underline{a}^{\prime} \theta_{i}\left(f_{0}, f_{n}\right)=\frac{1}{\sqrt{N}}\left[1, e^{-j \varphi\left(\theta_{i}, \hat{\theta}, f_{0}, f_{n}\right)}, \ldots, e^{-j(N-1) \varphi\left(\theta_{i}, \hat{\theta}, f_{0}, f_{n}\right)}\right]^{T}
$$

avec:

$$
\varphi\left(\theta_{i}, \hat{\theta}, f_{0}, f_{n}\right)=2 \pi \frac{d}{c} f_{0} \sin \hat{\theta}+2 \pi \frac{d}{c} f_{n}\left(\sin \theta_{i}-\sin \hat{\theta}\right)
$$

En posant : $f_{n}=f_{0}+\Delta f_{n} \quad\left(\Delta f_{n}\right.$ s'exprime en fonction de $\left.1 / T\right)$



Figure 1 : Exemple de spectre large bande

$\varphi\left(\theta_{i}, \hat{\theta}, f_{0}, f_{n}\right)=2 \pi \frac{d}{c} f_{0} \sin \theta_{i}+2 \pi \frac{d}{c} \Delta f_{n}\left(\sin \theta_{i}-\sin \hat{\theta}\right)$

$\varphi\left(\theta_{i}, \hat{\theta}, f_{0}, f_{n}\right)=\varphi\left(\theta_{i}, f_{0}\right)+\epsilon\left(\theta_{i}, \hat{\theta}, \Delta f_{n}\right)$

Donc après focalisation, le déphasage entre capteurs est la somme du déphasage lié à l'azimut $\theta_{i}$ à la fréquence $f_{0}$ et d'un terme $\in\left(\theta_{i}, \hat{\theta}, \Delta f_{n}\right)$. Ce terme ramené à la fréquence $f_{0}$ s'interprétera comme une erreur $\Delta \theta_{\mathrm{i}}$ sur l'azimut $\theta_{\mathrm{i}}$.

Soit $\varphi\left(\theta_{i}, \hat{\theta}, f_{0}, f_{n}\right)=\varphi\left(\theta_{i}+\Delta \theta_{i}, f_{0}\right)$

Pour des valeurs de $\Delta \theta_{i}$ faibles, on a :

$2 \pi f_{0} \frac{d}{c} \sin \left(\theta_{i}+\Delta \theta_{i}\right)=2 \pi f_{0} \frac{d}{c} \sin \theta_{i}+2 \pi f_{0} \frac{d}{c} \cos \theta_{i}$

De (1) et (2) on obtient :

$$
\Delta \theta_{i}=\frac{\epsilon\left(\theta_{i}, \hat{\theta}, \Delta f_{n}\right)}{2 \pi f_{0} \frac{d}{c} \cos \theta_{i}}=\frac{\Delta f_{n}}{f_{0}} \frac{\sin \theta_{i}-\sin \hat{\theta}}{\cos \theta_{i}} \quad \text { avec } \theta i \neq \pi / 2+k \pi \text { et } f_{0} \neq 0 \mathrm{~Hz}
$$

L'expression (3) montre que l'erreur $\Delta \theta_{i}$ est fonction de la fréquence de focalisation $f_{0}$, de la largeur de la bande et de l'initialisation $\hat{\theta}$.

\section{3-1 Choix de la fréquence de focalisation}

Afin d'étudier l'influence de la fréquence de focalisation sur le biais d'estimation des azimuts des sources, nous supposons que la largeur de la bande est fixée à $L=\left[f_{1}, f_{M}\right]$ et nous considérons les cas limites de $\mathrm{f}_{0}$ :

a) $f_{0}=f_{1}$ (fréquence minimale de la bande)

b) $f_{0}=f_{c}$ (fréquence centrale de la bande) 


\section{4-1 Biais dû à l'initialisation}

Afin de chiffrer l'importance du choix de la direction initiale, nous avons traité le cas d'une seule source placée à l'azimut $10^{\circ}$ et de largeur de bande égale à $40 \mathrm{~Hz}(\mathrm{~L}=90$ à $130 \mathrm{~Hz})$. Le nombre de capteurs alignés et équidistants de $c / 2 \mathrm{f}_{\mathrm{M}}$ est égal à 6 . Les résultats obtenus pour différentes valeurs de la direction initiale sont regroupés dans le tableau présenté figure 3.

\begin{tabular}{|c|c|}
\hline$\theta_{0}$ & $|\theta-\hat{\theta}|$ \\
\hline$-30^{\circ}$ & $0.30^{\circ}$ \\
\hline$-20^{\circ}$ & $0.23^{\circ}$ \\
\hline$-10^{\circ}$ & $0.15^{\circ}$ \\
\hline $0^{\circ}$ & $0.07^{\circ}$ \\
\hline $10^{\circ}$ & $0.00^{\circ}$ \\
\hline $20^{\circ}$ & $0.07^{\circ}$ \\
\hline $30^{\circ}$ & $0.14^{\circ}$ \\
\hline $40^{\circ}$ & $0.20^{\circ}$ \\
\hline $50^{\circ}$ & $0.27^{\circ}$ \\
\hline
\end{tabular}

Figure 3: variations du biais en fonction de la direction initiale utilisée pour la construction des opérateurs de focalisation.

En analysant le tableau de la figure 3, nous constatons que plus la valeur de la direction initiale s'éloigne de la vraie valeur $\left(10^{\circ}\right)$, plus l'erreur augmente. L'erreur est alors nulle lorsque la direction initiale est confondue à la vraie direction. Ceci est tout à fait conforme aux résultats théoriques obtenus.

\section{4-2 Importance du choix de la fréquence de focalisation}

Deux sources totalement cohérentes de même bande fréquentielle de largeur égale à $40 \mathrm{~Hz}$ $\left(90 \mathrm{~Hz}\right.$ à $130 \mathrm{~Hz}$ ) sont placées aux azimuts $80^{\circ}$ et $84^{\circ}$. Le rapport signal sur bruit pour chaque source est de $10 \mathrm{~dB}$. Le nombre de capteurs, alignés et équidistants de $d=c / 2 f_{M}$ est égal à 10 . La direction de focalisation est choisie à $82^{\circ}$. Les résultats obtenus (figure 4), montrent que pour les deux sources, le biais est minimal pour la fréquence de focalisation égale à la fréquence centrale de la bande $f_{0}=110 \mathrm{~Hz}$.

\begin{tabular}{|c|l|l|}
\hline$f_{0}(\mathrm{~Hz})$ & $\left|\theta_{1}-\hat{\theta}_{1}\right|$ & $\left|\theta_{2}-\hat{\theta}_{2}\right|$ \\
\hline 90 & $0.41^{\circ}$ & $0.97^{\circ}$ \\
\hline 100 & $0.21^{\circ}$ & $0.40^{\circ}$ \\
\hline 110 & $0.04^{\circ}$ & $0.05^{\circ}$ \\
\hline 120 & $0.09^{\circ}$ & $0.18^{\circ}$ \\
\hline 130 & $0.22^{\circ}$ & $0.37^{\circ}$ \\
\hline
\end{tabular}

Figure 4 : Biais d'estimation des azimuts des sources en fonction de la fréquence de focalisation 
c) $f_{0}=f_{M}$ (fréquence maximale de la bande)

Dans les trois cas considérés (figure 2), les variations de l'onde focalisée occupent la même bande sur l'axe des fréquences spatiales. La moyenne réalisée sur la bande conduit à une erreur globale minimale voire nulle pour $\mathrm{f}_{0}=\mathrm{f}_{\mathrm{C}}$.
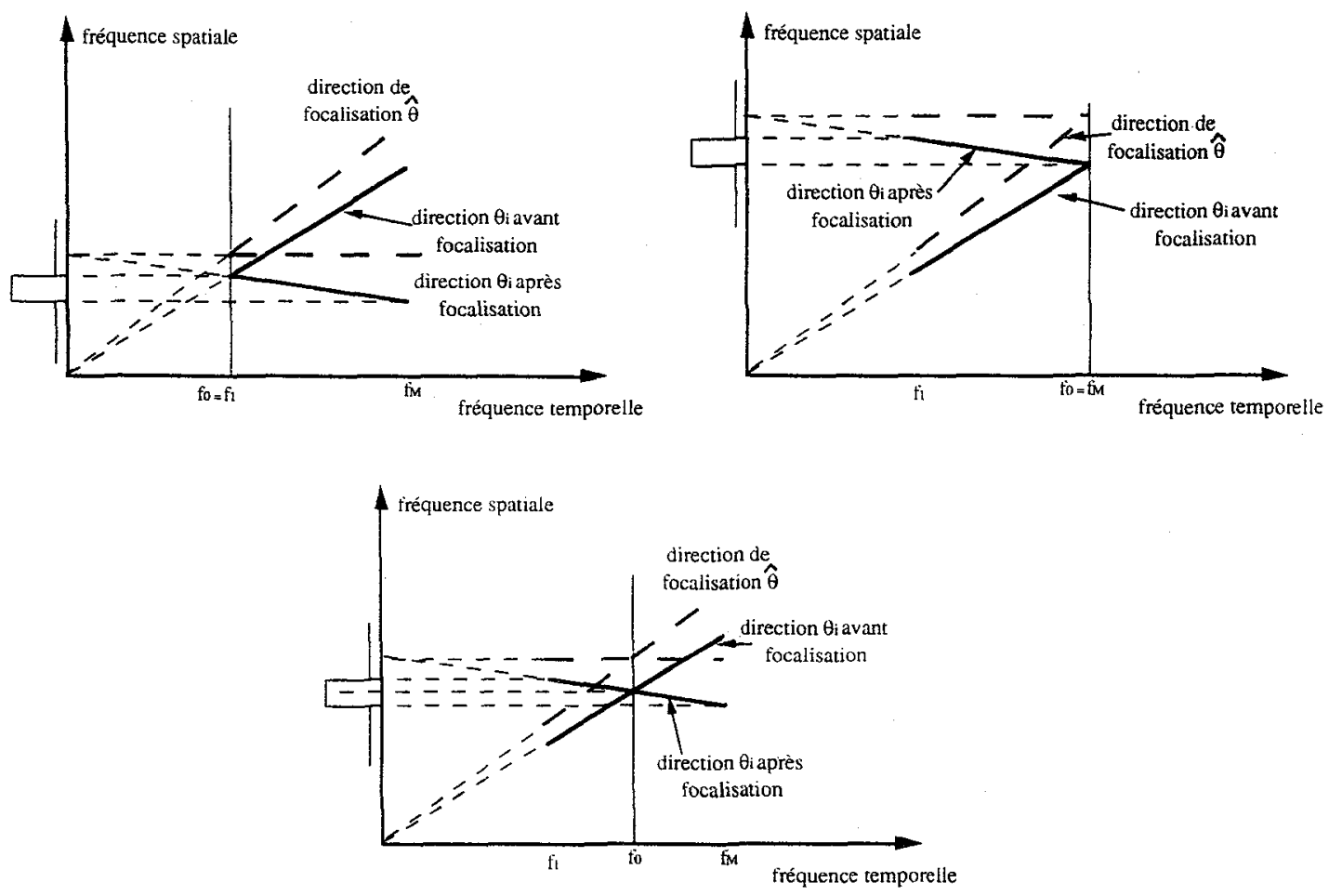

Figure 2: Variations de l'onde focalisée en fonction de fo

\section{3-2 Choix de la largeur de la bande d'analyse :}

Parmi les intérêts de la focalisation, il y a le fait que l'on peut résoudre des sources cohérentes [2], [3] sans aucune condition supplémentaire sur le nombre de capteurs à utiliser, contrairement à ce qui se passe lors du lissage spatial. Cet avantage s'obtient par le biais de la moyenne réalisée sur les différentes fréquences d'analyse.

Pour décorréler les sources, en général il est préférable de moyenner beaucoup (choisir $M$ grand). Pour minimiser l'erreur $\Delta \theta_{\mathbf{i}}$ après focalisation, il faut choisir $\mathbf{M}$ petit. Nous constatons alors qu'un compromis doit être fait entre la décorrélation et le biais. Signalons qu'un taux de corrélation de $80 \%$, donne en pratique des résultats acceptables. Ceci nous permet alors de trouver plus facilement un compromis entre biais et corrélation.

\section{4 - APPLICATION A DES SIGNAUX SIMULES}

Afin de confirmer les résultats théoriques présentés ci-dessus, nous avons réalisé de façon synthétique quelques exemples proches de la réalité physique. 


\section{4-3 Largeur de la bande à analyser}

Nous avons simulé deux situations:

- dans un premier temps, une source est placée à $80^{\circ}$, sa bande de fréquences totale est égale à $60 \mathrm{~Hz}(60$ à $120 \mathrm{~Hz})$,

- dans un deuxième temps, nous avons augmenté la largeur de la bande qui est passée de $60 \mathrm{~Hz}$ à $90 \mathrm{~Hz}(45$ à $135 \mathrm{~Hz})$.

Dans les deux cas, le rapport signal sur bruit et de $10 \mathrm{~dB}$, le nombre de capteurs alignés et équidistants de $\mathrm{c} / 2 \mathrm{f}_{\mathrm{M}}$ est égal à 6 . La direction de focalisation est $82^{\circ}$. Pour le premier cas, la fréquence de focalisation est de $60 \mathrm{~Hz}$ et pour le deuxième cas, c'est la fréquence centrale qui est choisie.

Nous regroupons sur les tableaux 1 et 2 , figure 5, les variations du biais en fonction de la largeur de la bande $L$, comparée à la fréquence centrale $f_{c}$. Ces résultats montrent que plus la bande d'analyse est grande, plus le biais d'estimation de l'azimut est important.

La comparaison des résultats présentés dans les deux tableaux 1 et 2 montre que le choix de la fréquence de focalisation est plus important que le choix de la largeur de la bande à analyser si l'on veut minimiser le biais.

Compte-tenu des différents résultats obtenus concernant le biais, nous pouvons classer les paramètres de focalisation dans l'ordre d'importance suivant :

-angles de focalisation,

-fréquence de focalisation,

-largeur de la bande à analyser.

\begin{tabular}{|c|c|}
\hline $\mathrm{L} / \mathrm{f}_{0}$ & $|\theta-\hat{\theta}|$ \\
\hline $100 \%$ & $0.93^{\circ}$ \\
\hline $80 \%$ & $0.71^{\circ}$ \\
\hline $60 \%$ & $0.46^{\circ}$ \\
\hline $40 \%$ & $0.23^{\circ}$ \\
\hline $20 \%$ & $0.16^{\circ}$ \\
\hline
\end{tabular}

Tableau 1

\begin{tabular}{|c|c|}
\hline $\mathrm{L} / \mathrm{f}_{0}$ & $|\theta-\hat{\theta}|$ \\
\hline $100 \%$ & $0.12^{\circ}$ \\
\hline $80 \%$ & $0.02^{\circ}$ \\
\hline $60 \%$ & $0.01^{\circ}$ \\
\hline $40 \%$ & $0.00^{\circ}$ \\
\hline $20 \%$ & $0.00^{\circ}$ \\
\hline
\end{tabular}

Tableau 2

Figure 5 : Variations du biais d'estimation de l'azimut en fonction de la largeur de la bande d'analyse

\section{5 - APPLICATION A DES SIGNAUX EXPERIMENTAUX}

Nous avons pu mettre en oeuvre les méthodes de localisation large bande basées sur l'utilisation d'opérateurs de focalisation sur des données expérimentales. Pour cela nous avons utilisé les signaux provenant d'une campagne en mer [1] organisée par le GERDSM. La réception des signaux est faite sur une grande antenne horizontale. Nous considérons la bande de fréquence des signaux à traiter, définie par la bande du signal d'une source coopérative connue. Cette bande est de $20 \mathrm{~Hz}$ située autour de $82 \mathrm{~Hz}$.

A l'aide de la tranformée de Fourier rapide, la bande du signal est divisée en 100 fréquences, à chaque fréquence la matrice spectrale est estimée à partir des signaux reçus sur dix capteurs ( 1 à 10) de l'antenne. Ces matrices sont estimées en utilisant la méthode du périodogramme lissée avec un $\mathrm{B}_{\mathrm{e}} \mathrm{T}=20$. 
- Afin d'estimer le nombre de sources $P$, les critères AIC-MDL [1] ont été appliqués à la fréquence de focalisation $f_{0}=82 \mathrm{~Hz}$. Les deux critères ont conduit au même résultat $P=2$.

- Pour estimer les azimuts des sources détectées, nous avons utilisé les deux méthodes principales, pour la mise en oeuvre de la méthode à sous-espace signal cohérent, nous avons utilisé différents opérateurs de focalisation :

La figure 6 montre les différents résultats obtenus pour l'analyse à toutes les fréquences. La figure 7 donne une représentation sous forme de courbes de niveaux de ces résultats. Nous constatons des fluctuations d'azimuts autour des valeurs moyennes $: 3^{\circ}$ et $7^{\circ}$.

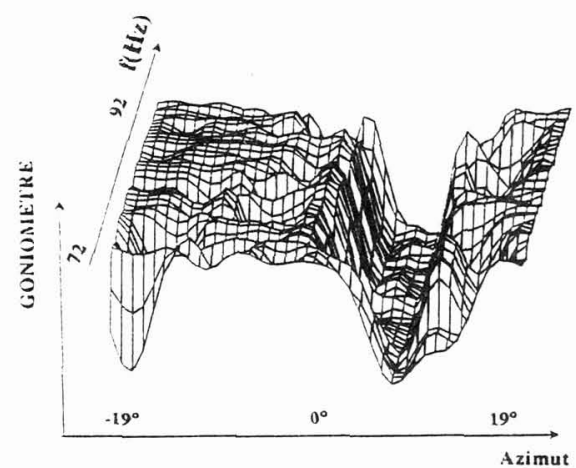

Figure 6 : Analyse de la bande, fréquence par fréquence. (avec un pas de $0.5 \mathrm{~Hz}$ )

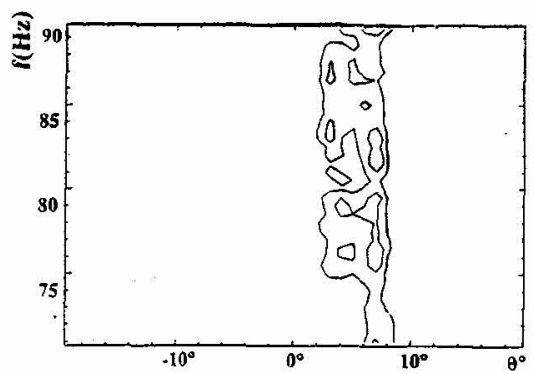

Figure 7 : Courbes de niveaux

Dans un premier temps, l'opérateur de focalisation préconisé par Wang et Kaveh est utilisé pour effectuer la focalisation, les directions initiales choisies sont $\theta_{1}=3^{\circ}$ et $\theta_{2}=7^{\circ}$. Comme précédemment, nous présentons (figure 8 ) les résultats obtenus, en décrivant toute la bande d'analyse, les courbes de niveau correspondantes sont présentées figure 9.

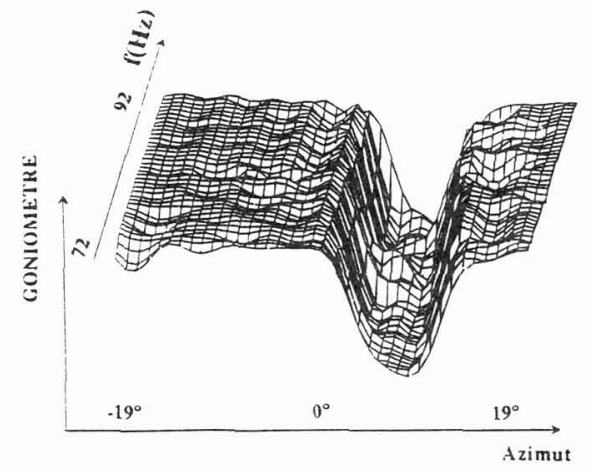

Figure 8 : Analyse fréquence par fréquence après focalisation par la méthode de Wang.



Figure 9 : Courbes de niveaux

Dans un deuxième temps, nous mettons en oeuvre l'opérateur de focalisation utilisant les vecteurs propres des matrices interspectrales; nous montrons (figures 10 et 11) les résultats obtenus. 


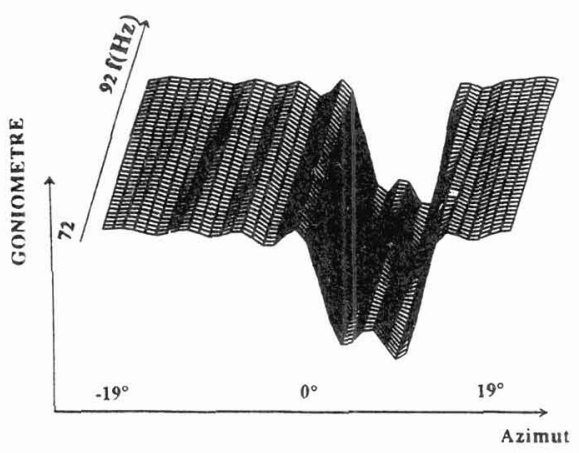

Figure 10 : Analyse fréquence par fréquence après focalisation par la méthode proposée.

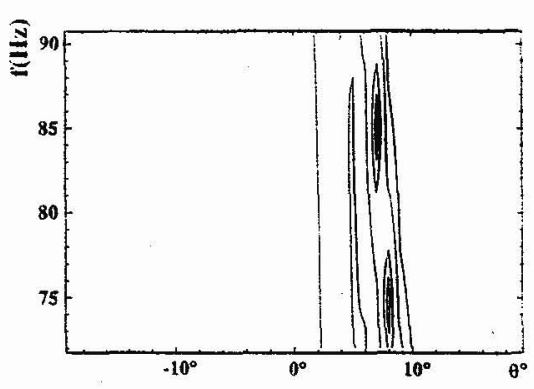

Figure 11 : Courbes de niveaux

Nous constatons, après focalisation que la dispersion des azimuts autour des valeurs moyennes est beaucoup plut petite, ce qui permet de conclure à une robustesse au bruit de la méthode de focalisation. Cette amélioration est due au moyennage cohérent effectué sur l'ensemble des fréquences de la bande.

\section{CONCLUSION}

Cette étude qui traite de la localisation de sources rayonnantes large bande a permis de donner des règles de contrôle des paramètres de focalisation lors de l'utilisation des méthodes de sous espace signal cohérent. Pour cela nous avons chiffré le biais d'estimation des azimuts en fonction de ces paramètres à savoir la fréquence, les azimuts de focalisation et la largeur de la bande à analyser. En utilisant les signaux synthétiques nous avons confirmé les différents résultats théoriques et nous avons fixé leurs valeurs optimales à utiliser en pratique. Nous avons pu classer ces paramètres par ordre d'importance.

Nous avons proposé un nouvel opérateur de focalisation ne nécessitant pas une connaissance a priori des azimuts des sources. L'application des diverses méthodes de traitement d'antenne à large bande sur des données expérimentales nous a permis de comparer leurs performances et de conclure que les méthodes utilisant le sous espace signal cohérent sont plus robustes au bruit et que l'opérateur proposé est aussi performant que les autres.

Notons que ces méthodes de focalisation permettent de traiter le problème de sources totalement cohérentes sans aucune condition supplémentaire concernant le nombre de capteurs.

\section{REFERENCES}

[1] S. BOURENNANE, B. FAURE, J.L. LACOUME, Traitement d'antenne pour des sources bande large. Revue Ann. télécomm., à paraître.

[2] H. WANG, M. KAVEH, Coherent signal subspace processing for the detection and estimation of angles of arrival of multiple wideband sources. IEEE ASSP-33, p.823-831, Aug. 1985.

[3] A.K. SHAW, R. KUMARESAN, Estimation of angles of arrivals of broadband signals. Proc. IEEE ICASSP'87, p.2296-2299, 1987.

[4] H. HUNG, M. KAVEH, Focusing matrices for coherent signal processing. IEEE ASSP, Vol.36, p.1272-1281, Aug.1988.

Remerciements : Cette étude à été conduite avec le soutien de la D.C.N. 